\title{
ERRATUM
}

\section{Saxagliptin: a New DPP-4 Inhibitor for the Treatment of Type 2 Diabetes Mellitus}

Abd A. Tahrani · Milan K. Piya $\cdot$ Anthony H. Barnett

(c) Springer Healthcare Communications 2009

In the review by Abd Tahrani and colleagues entitled 'Saxagliptin: a New DPP-4 Inhibitor for the Treatment of Type 2 Diabetes Mellitus', published in Advances in Therapy 2009 Mar;26(3):249262 , the section on page 252 that reads:

"Saxagliptin demonstrates greater specificity for DPP-4 than for either the DPP-8 or DPP-9 enzymes (400- and 75 -fold, respectively). ${ }^{46}$ The active metabolite of saxagliptin (BMS-510849) is two-fold less potent than the parent. Both saxagliptin and its metabolite are highly selective ( $>4000$-fold) for the inhibition of DPP-4 compared with a range of other proteases (selectivity of sitagliptin and vildagliptin for DPP-4 is $>2600$ and 32-250-fold, respectively, compared with DPP-8/9). ${ }^{4 \prime \prime}$

could be open to misinterpretation, and should have appeared as follows:

"Saxagliptin demonstrates greater selectivity for DPP-4 than for either the DPP-8 or DPP-9 enzymes (400- and 75-fold, respectively). ${ }^{46}$ The active metabolite of saxagliptin (BMS-510849) is two-fold less potent than the parent. Selectivity of sitagliptin and vildagliptin for DPP-4 is $>2600$ and 32-250-fold greater, respectively, compared with DPP-8/9.44 Both saxagliptin and BMS-510849 are also highly selective for inhibition of DPP-4 compared with a large panel of other proteases tested (>4000-fold)." 